\title{
Correction to: Mitigating Disparity in Healthcare Resources Between Countries for Management of Hereditary Angioedema
}

\author{
Ankur Kumar Jindal ${ }^{1} \cdot$ Avner Reshef $^{2} \cdot$ Hilary Longhurst ${ }^{3,4,5}$. \\ GEHM workgroup (Global Equity in H. A. E. Management)
}

Published online: 1 July 2021

(c) The Author(s) 2021

\section{Correction to: Clinical Reviews in Allergy \& Immunology: https://doi.org/10.1007/s12016-021-08854-5}

The original version of this article unfortunately contained a mistake. We need to add a contributor to Table 1, who contributed to the paper, in particular the data collection and whose name was left off the final list of contributors. The corrected Table 1 is given below. The original article has been corrected.

The original article can be found online at https://doi.org/10.1007/ s12016-021-08854-5.

Hilary Longhurst

hlonghurst@doctors.org.uk

1 Allergy Immunology Unit, Department of Paediatrics, Advanced Paediatrics Centre, Postgraduate Institute of Medical Education and Research, Chandigarh, India

2 Allergy Immunology \& Angioedema Center, Barzilai University Medical Center, Ashkelon, Israel

3 Auckland District Health Board, Auckland, New Zealand

4 Department of Allergy and Immunology, University College Hospitals, London, England

5 Department of Medicine, University of Auckland, Auckland, New Zealand 
Table 1 List of the Global Equity in HAE Management (GEHM) workgroup participants

\begin{tabular}{|c|c|c|c|c|c|}
\hline & Country & Name & & Affiliation & Mail \\
\hline 1 & Austria & Aberer & Werner & $\begin{array}{l}\text { Medical University of Graz, Graz, } \\
\text { Austria }\end{array}$ & werner.aberer@medunigraz.at \\
\hline 2 & Canada & Betchel & Stephen & $\begin{array}{l}\text { Univ. of Toronto, St. Michael's Hospi- } \\
\text { tal, Toronto, Canada }\end{array}$ & Stephen.Betschel@unityhealth.to \\
\hline 3 & Germany & $\begin{array}{l}\text { Bork } \\
\text { Aygören-Pürsün } \\
\text { Maurer } \\
\text { Magerl }\end{array}$ & $\begin{array}{l}\text { Konrad } \\
\text { Emel } \\
\text { Marcus } \\
\text { Markus }\end{array}$ & $\begin{array}{l}\text { Universitäts-Hautklinik, Dermatology, } \\
\text { Mainz, Germany } \\
\text { Goethe-Universität, Frankfurt am } \\
\text { Main, Germany } \\
\text { Charité - Universitätsmedizin Berlin, } \\
\text { Berlin, Germany } \\
\text { Charité - Universitätsmedizin Berlin, } \\
\text { Berlin, Germany }\end{array}$ & $\begin{array}{l}\text { konrad.bork@unimedizin-mainz.de } \\
\text { aygoeren@em.uni-frankfurt.de } \\
\text { marcus.maurer@charite.de } \\
\text { markus.magerl@charite.de }\end{array}$ \\
\hline 4 & France & Bouillet & Laurence & $\begin{array}{l}\text { Chercheur à Université Grenoble } \\
\text { Alpes, Grenoble, France }\end{array}$ & LBouillet@chu-grenoble.fr \\
\hline 5 & Denmark & Bygum & Anette & $\begin{array}{l}\text { Odense Universitetshospital, Odense, } \\
\text { Denmark }\end{array}$ & Anette.Bygum@rsyd.dk \\
\hline 6 & Spain & Caballero & Teresa & $\begin{array}{l}\text { Hospital Universitario La Paz, } \\
\text { Madrid, Spain }\end{array}$ & tercaballero@gmail.com \\
\hline 7 & Italy & Cancian & Mauro & $\begin{array}{l}\text { University Hospital of Padova, } \\
\text { Padova, Italy }\end{array}$ & mcancian@unipd.it \\
\hline 8 & Hungary & Farkas & Henriette & $\begin{array}{l}\text { Angioedema Ctr, Semmelweis Univer- } \\
\text { sity, Budapest, Hungary }\end{array}$ & $\begin{array}{l}\text { farkas.henriette@med.semmelweis- } \\
\text { univ.hu }\end{array}$ \\
\hline 9 & North Macedonia & Grivcheva-Panovska & Vesna & $\begin{array}{l}\text { University Sts Cyril and Methodius } \\
\text { Skopje, North Macedonia }\end{array}$ & $\begin{array}{l}\text { vesna_grivcheva_panovska@yahoo. } \\
\text { com }\end{array}$ \\
\hline 10 & Brazil & Grumach & Anete & $\begin{array}{l}\text { Ctr for Rare Diseases, Faculdade de } \\
\text { Medicina, São Paulo, Brazil }\end{array}$ & anete@grumach.com \\
\hline 11 & Turkey & Gulbahar & Okan & Ege Üniversitesi, Izmir, Turkey & okan.gulbahar@yahoo.com \\
\hline 12 & Japan & Hide & Michihiro & $\begin{array}{l}\text { Dept of Dermatology, Hiroshima } \\
\text { Univ. Hiroshima, Japan }\end{array}$ & ed1h-w1de-road@hiroshima-u.ac.jp \\
\hline 13 & India & $\begin{array}{l}\text { Jindal } \\
\text { Singh }\end{array}$ & $\begin{array}{l}\text { Ankur } \\
\text { Surjit }\end{array}$ & $\begin{array}{l}\text { Postgraduate Institute of Medical } \\
\text { Education and Research, Chandi- } \\
\text { garh, India } \\
\text { Postgraduate Institute of Medical } \\
\text { Education and Research, Chandi- } \\
\text { garh, India }\end{array}$ & $\begin{array}{l}\text { ankurjindal11@gmail.com } \\
\text { surjitsinghpgi@ rediffmail.com }\end{array}$ \\
\hline 14 & Bangladesh & Jindal & Ankur & $\begin{array}{l}\text { Postgraduate Institute of Medical } \\
\text { Education and Research, Chandi- } \\
\text { garh, India }\end{array}$ & ankurjindal11@gmail.com \\
\hline 15 & South Korea & Kang & Hye-Ryun & $\begin{array}{l}\text { Seoul National University Hospital, } \\
\text { Seoul, South Korea }\end{array}$ & helenmed@snu.ac.kr \\
\hline 16 & Israel & $\begin{array}{l}\text { Reshef } \\
\text { Kessel }\end{array}$ & $\begin{array}{l}\text { Avner } \\
\text { Aharon }\end{array}$ & $\begin{array}{l}\text { Barzilai University Medical Ctr, } \\
\text { Ashkelon, Israel } \\
\text { Bnay-Zion Med Ctr, Technion Medi- } \\
\text { cal School, Haifa, Israel }\end{array}$ & $\begin{array}{l}\text { avnerre@bmc.gov.il } \\
\text { aharon.kessel@b-zion.org.il }\end{array}$ \\
\hline 17 & United Kingdom & Longhurst & Hilary & $\begin{array}{l}\text { UCLH, London, UK } \\
\text { Department of Medicine, University } \\
\text { of Auckland, New Zealand }\end{array}$ & hlonghurst@doctors.org.uk \\
\hline 18 & New Zealand & $\begin{array}{l}\text { Lindsay } \\
\text { Jordan } \\
\text { Ameratunga }\end{array}$ & $\begin{array}{l}\text { Karen Anthony } \\
\text { Rohan }\end{array}$ & $\begin{array}{l}\text { Auckland District Health Board } \\
\text { Auckland District Health Board } \\
\text { Department of Molecular Medicine } \\
\text { and Pathology Faculty of Medical } \\
\text { and Health Sciences, University of } \\
\text { Auckland }\end{array}$ & $\begin{array}{l}\text { KLindsay@ adhb.govt.nz } \\
\text { AnthonyJ@ adhb.govt.nz } \\
\text { rame001@aucklanduni.ac.nz }\end{array}$ \\
\hline
\end{tabular}


Table 1 (continued)

\begin{tabular}{|c|c|c|c|c|c|}
\hline & Country & Name & & Affiliation & Mail \\
\hline 19 & USA & $\begin{array}{l}\text { Lumry } \\
\text { Bernstein } \\
\text { Craig } \\
\text { Riedl } \\
\text { Levy }\end{array}$ & $\begin{array}{l}\text { William } \\
\text { Jonathan } \\
\text { Timothy } \\
\text { Marc } \\
\text { Don }\end{array}$ & $\begin{array}{l}\text { Allergy \& Immunology Assoc., Dallas } \\
\text { TX, USA } \\
\text { Univ. Cincinnati, Div. of Immunology, } \\
\text { Cincinnati OH, USA } \\
\text { Penn State University Hershey, PA, } \\
\text { USA } \\
\text { US HAEA Angioedema Ctr, Univ. of } \\
\text { California, San Diego CA USA } \\
\text { University of California at Irvine, } \\
\text { Irvine, CA, USA }\end{array}$ & $\begin{array}{l}\text { Lumrymd@me.com } \\
\text { BERNSTJA@ucmail.uc.edu } \\
\text { tcraig@pennstatehealth.psu.edu } \\
\text { mriedl@ health.ucsd.edu } \\
\text { DLevy1@uci.edu }\end{array}$ \\
\hline 20 & Argentina & Malbran & Alejandro & $\begin{array}{l}\text { Asociación Argentina de AH, Buenos } \\
\text { Aires, Argentina }\end{array}$ & amalbran31@hotmail.com \\
\hline 21 & Greece & $\begin{array}{l}\text { Germenis } \\
\text { Psarros }\end{array}$ & $\begin{array}{l}\text { Anastasios } \\
\text { Fotis }\end{array}$ & $\begin{array}{l}\text { School of Medicine, University of } \\
\text { Thessaly, Larissa, Greece } \\
\text { Greek Navy Hospital, Athens, Greece }\end{array}$ & $\begin{array}{l}\text { agermen@med.uth.gr } \\
\text { psarros@ allergy.gr }\end{array}$ \\
\hline 22 & Poland & $\begin{array}{l}\text { Stobiecki } \\
\text { Porebski }\end{array}$ & $\begin{array}{l}\text { Marcin } \\
\text { Grzegorz }\end{array}$ & $\begin{array}{l}\text { Jagelonian University, Krakow, Poland } \\
\text { Department of Clinical and Environ- } \\
\text { mental Allergology, Jagiellonian } \\
\text { University Medical College ul. } \\
\text { Botaniczna 3, Krakow, Poland }\end{array}$ & $\begin{array}{l}\text { marcin.stobiecki@uj.edu.pl } \\
\text { g.porebski@uj.edu.pl }\end{array}$ \\
\hline 23 & Bulgaria & Valerieva & Anna & $\begin{array}{l}\text { University Hospital "Alexandrovska", } \\
\text { Sofia, Bulgaria }\end{array}$ & anna.valerieva@gmail.com \\
\hline $\begin{array}{l}24 \\
25 \\
26\end{array}$ & $\begin{array}{l}\text { Australia } \\
\text { Indonesia } \\
\text { Hong Kong }\end{array}$ & Wardman & Fiona & $\begin{array}{l}\text { HAE International (HAEi), Chief } \\
\text { Regional Patient Advocate and } \\
\text { Regional Patient Advocate, Asia } \\
\text { Pacific }\end{array}$ & f.wardman@haei.org \\
\hline 27 & Singapore & $\begin{array}{l}\text { Wardman } \\
\text { Zhong }\end{array}$ & $\begin{array}{l}\text { Fiona } \\
\text { Youjia }\end{array}$ & $\begin{array}{l}\text { HAE International (HAEi), Chief } \\
\text { Regional Patient Advocate and } \\
\text { Regional Patient Advocate, Asia } \\
\text { Pacific } \\
\text { National University Hospital, Singa- } \\
\text { pore }\end{array}$ & $\begin{array}{l}\text { f.wardman@haei.org } \\
\text { youjia_zhong@nuhs.edu.sg }\end{array}$ \\
\hline 28 & Switzerland & Weber & Christina & $\begin{array}{l}\text { Allergiestation, Universitätsspital } \\
\text { Zürich, Switzerland }\end{array}$ & weber@chinderarztpraxis.ch \\
\hline
\end{tabular}

Open Access This article is licensed under a Creative Commons Attribution 4.0 International License, which permits use, sharing, adaptation, distribution and reproduction in any medium or format, as long as you give appropriate credit to the original author(s) and the source, provide a link to the Creative Commons licence, and indicate if changes were made. The images or other third party material in this article are included in the article's Creative Commons licence, unless indicated otherwise in a credit line to the material. If material is not included in the article's Creative Commons licence and your intended use is not permitted by statutory regulation or exceeds the permitted use, you will need to obtain permission directly from the copyright holder. To view a copy of this licence, visit http://creativecommons.org/licenses/by/4.0/.

Publisher's Note Springer Nature remains neutral with regard to jurisdictional claims in published maps and institutional affiliations. 